\title{
Physical and cognitive dimensions in stimulus comparison
}

\author{
W. R. GARNER \\ Yale University, New Haven, Connecticut \\ PETER PODGORNY \\ University of Southern California, Los Angeles, California \\ and \\ ELLEN M. FRASCA \\ Yale University, New Haven, Connecticut
}

\begin{abstract}
Two experiments were carried out using a same-different task with sets of four stimuli varying orthogonally in three dimensions. Sameness was defined by each of the three dimensions in turn, as well as by physical identity. Two types of dimensions, physical and cognitive, were studied. In Experiment 1, the numerals 6, 10, VI, and X, which vary in Length, System, and Name, were used. With simultaneous presentation, order of difficulty was from the physical dimension of Length to the cognitive dimension of Name. While overall difficulty was related to dimensional discriminability, internal evidence suggested that Name was not a dimension in the same sense as Length is, and that some stimulus pairs were simply easier to process than others, regardless of the response required (e.g., short stimulus pairs and Arabic numerals). With sequential presentation, Name was processed as fast as System, due largely to the fact that much faster responding occurred when an Arabic numeral was the second stimulus. Thus, with sequential presentation, Name provides a processing mechanism not provided by physical dimensions. In Experiment 2, the numerals 3, 4, 6, and 7, which vary in Magnitude, Oddness, and Curvilinearity, were used. The cognitive dimension of Magnitude was processed most rapidly, and numerical distance between pairs of numbers dominated the results for "same" responses, regardless of the sameness rule used. Again, evidence was found for fast processing of some stimulus pairs (e.g., 34 ), regardless of the response required. Overall, these experiments are interpreted as indicating that cognitive factors such as stimulus familiarity may override aspects of physical discriminability with many dimensions and stimuli, that, even though a cognitive dimension can be used to generate a logically proper set of stimuli, it does not necessarily act as other, more physical dimensions do, and that clarification of the functional role of a dimension is more important than attempts to locate stages.
\end{abstract}

Stimulus comparison techniques such as the samedifferent task have been used quite commonly to establish levels or hierarchies of processing, starting with the Posner and Mitchell (1967) research, with more recent research well reviewed in Posner (1978). The most frequently used stimulus materials have been visual letters, with the rule for sameness varied to manipulate the presumptive level of processing. To illustrate, in the Posner and Mitchell (1967) experiments, upper- and lowercase letters were used,

This research was supported by Grant MH 14229 from the National Institute of Mental Health to Yale University. Carolyn Paul acted as experimenter for part of Experiment 1, did much of the data analysis, and assisted in other aspects of the research. Requests for reprints should be sent to W. R. Garner, Department of Psychology, Box 11-A Yale Station, New Haven, Connecticut 06520 . and sameness was defined for the subjects in terms of physical identity (e.g., AA, aa, BB), of having the same name (e.g., $A a, B b)$, or of being both vowels or both consonants (e.g., AE, BT). The finding, by now common, is that it takes longer to decide that two letters are both vowels or both consonants than to decide that they have the same name, and that the latter decision in turn takes longer than it does to decide that two letters are physically identical.

Despite the consistent nature of results in such experiments, and the rather natural interpretation that the differences in reaction time (RT) indicate differences in levels of processing, there has been an awkward confounding factor in these experiments: When the rule for sameness is changed from one presumptive level to another, there is an increase in the number of stimuli that satisfy the definition of 
sameness; that is, the class inclusions become larger. Thus, with physical identity as the rule for sameness, only one stimulus satisfies the sameness requirement for any given stimulus, but with same name as the rule for sameness, two stimulus alternatives satisfy the requirement for sameness. Similarly, when the rule for sameness is extended so that all vowels or all consonants are to be called "same," the number of stimulus alternatives has been increased still further. An increase in RT would occur, however, simply with an increase in the number of alternatives satisfying the definition of sameness, even for stimuli that do not carry any implication of differences in levels of processing. If, for example, eight random shapes were used as stimuli, none with obvious name or any other association, an experimental manipulation that increased the number of stimuli to which a particular response was to be given would increase RT, whether the experimental procedure involved a same-different task or simply a classification task (e.g., Gottwald \& Garner, 1972).

It is possible, however, to consider the different rules for sameness as dimensions that can be crossed to provide sets of stimuli generated from orthogonal dimensions, stimuli similar to those used in various classification tasks studied by Garner (1974) and Garner and Felfoldy (1970) to investigate the interaction between stimulus dimensions. For example, Garner (1974, p. 137, 1980) and Nickerson (1972) have pointed out that the four stimuli formed from a pair of upperand lowercase letters can be considered to be generated by two dimensions, name and case, and that a rule for sameness could be used such that sameness means same name or same case. A sameness rule of either name or case allows two stimulus alternatives to satisfy the definition of sameness, and, although that is more than the one stimulus that satisfies the definition of physical identity (thus maintaining the confounding problem), comparison between the two dimensions used as rules for sameness does not involve the confounding.

In fact, as will be demonstrated in these experiments, three different dimensions can be used to form a set of four stimuli by the use of a Latinsquare arrangement, and such an arrangement makes possible a comparison of three different potential levels of processing (i.e., dimensions) without the confounding of levels with number of stimulus alternatives. Such an arrangement is the limit with four stimuli, since there are just three ways in which four stimuli can be divided into two groups of two, and each of these three ways can be used to define sameness. As a purely logical matter, of course, it is not necessary that any of the three systems of grouping need be labeled with an identifiable dimension, and one could do experiments in which at least one of the groupings was, in effect, the biconditional classification of two dimensions. To illustrate with upper- and lowercase letters, four stimuli might be A, a, B, and $b$. Sameness could be defined by the three possible groupings: $\mathrm{A}$ a/ $\mathrm{B}$ b, $\mathrm{A} \mathrm{B} / \mathrm{a} \mathrm{b}$, or $\mathrm{A} \mathrm{b} / \mathrm{B}$ a (where the slash separates the pairs of stimuli that fall into the same class and that thus require a response of "same"). The first grouping is a sameness rule of name, the second is case, and the third is the biconditional classification of name and case.

\section{Physical and Cognitive Dimensions}

The fact that it is possible to consider variables such as names or other labels as dimensions, and thus to use experimental paradigms with these variables playing the same ostensible role as other variables more commonly considered to be dimensions (such as size, shape, color, etc.), does not ensure that these variables are perceived and processed as dimensions in the same way as the other variables. Therefore, one of the major reasons for using two or three variables, all of which play an equivalent role in terms of the logic of stimulus set construction and all of which have the same number of stimulus alternatives that satisfy the rule for sameness, is to provide comparisons between different types of stimulus dimension, as well as comparisons between dimensions of the same general type.

One major difference in the kinds of variables that can be and have been used in experiments of stimulus comparison is that between those dimensions for which there is an obvious and usually fairly simple physical property and those for which the physical property is less obvious or practically nonexistent, such as name equivalence, arbitrary ordering of numbers and letters of the alphabet, vowel-consonant equivalences, etc. The former type of dimension we shall call physical, and the latter type of dimension we shall call cognitive. The basic property of a physical dimension is that the dimension is an inherent physical property of stimuli that vary along it. Examples are height, length, intensity, size, color, and form. The basic property of a cognitive dimension is that the dimensional levels are arbitrary and must be learned before they can function as dimensions. Thus, the fact that $A$ is a letter that appears early in the alphabet and $Z$ is a letter that appears late is a cultural convention that must be learned and has no obvious physical property to justify its dimensionality. Also, the facts that $A$ and a have the same name and that $A$ and $E$ are both vowels are due to arbitrary conventions and must be learned before such dimensionality can be used in an information processing task.

The distinction between physical and cognitive dimensions is not a clear dichotomy, because stimuli can vary in the degree to which they differ on "obvious" physical dimensions. Furthermore, there is no way in which stimuli can be generated for use along cognitive dimensions without there being physical dif- 
ferences among the stimuli, since, if there were no physical differences, any information processing task requiring differential responding would be impossible. Thus, $\mathbf{A}$ and a have the same name, and that name is different from that assigned to $B$ and $b$, but the two pairs of stimuli do have physical differences upon which to base the appropriate responding. So the question as to whether cognitive dimensions can be defined in terms of physical properties is not the primary point; rather, it is whether the nature of the physical properties is reasonably obvious and could have been used without recourse to the learned cognitive labels.

This distinction is not unlike that which Broadbent (1970) made between stimulus sets and response sets in tasks of selective attention. Stimulus sets allow selective attention on the basis of rather obvious physical properties, whereas response sets allow selective attention on the basis of response categories. Garner (1976) pointed out, in discussing a paper by Keren (1976), that the distinction between stimulus set and response set is really a distinction between adequate and inadequate stimulus information. That is the distinction being made here: Physical dimensions are those on which the physical differences alone are adequate for carrying out the task, whereas cognitive dimensions are those on which the physical differences are by themselves inadequate for carrying out the task and some ascribed and learned property of the stimuli (which might be the response in an appropriate task) is necessary.

\section{Purpose of the Experiments}

The purpose of the present experiments was to explore with a same-different task the relation between physical and cognitive dimensions. The stimulus arrangement used three dimensions to define sets of four stimuli, thus allowing comparisons between three dimensions, all of which had the same number of stimulus alternatives that satisfied the sameness rule used. Two experiments were carried out with two different sets of stimuli, but in each experiment there were dimensions of a physical and of a cognitive type. A major concern of these experiments was whether physical dimensions were necessarily processed at a lower (i.e., more peripheral) level than cognitive dimensions. In addition, we were concerned with whether cognitive dimensions were processed in the same way as physical dimensions when they had the same structural role in the experimental design.

\section{GENERAL METHODS}

Two experiments were run, each with a different set of four stimuli. The first experiment used both a simultaneous and a sequential presentation of stimulus pairs, and the second experiment used only the simultaneous presentation. All other aspects of method were the same.

\section{Stimulus Presentation}

The stimuli were presented in a Scientific Prototype three-field tachistoscope, for which $12.7 \times 17.8 \mathrm{~cm}$ cards were used to display the stimuli. The stimuli were viewed binocularly at $127 \mathrm{~cm}$. The stimuli were black numerals on a white background. The background luminance was $5.0 \mathrm{fL}$.

Simultaneous presentation. The two stimuli of a pair were presented in the lower left and upper right quadrants of the stimulus field, off the diagonal, in order to prevent special properties of pairs, such as symmetries, from influencing response times. The pair was presented $500 \mathrm{msec}$ after initiation of a trial (during which time a blank field was exposed), and the pair of stimuli remained exposed for 1,000 msec regardless of the RT.

Sequential presentation. The first stimulus was presented during the $500-\mathrm{msec}$ interval after the initiation of a trial and was followed immediately by the second stimulus for $1,000 \mathrm{msec}$. The two stimuli never overlapped in time. The first stimulus was in the lower left quadrant, and the second stimulus was in the upper right quadrant.

\section{Procedure}

A single trial commenced when the subject pushed a button switch held in the left hand, at which time the blank interval occurred with simultaneous presentation and the first stimulus appeared with sequential presentation. The subject decided whether the pair of stimuli was the same or different by the appropriate rule for sameness and responded by moving a small switch lever mounted under the tachistoscope, to the right for "same" and to the left for "different." A computer recorded the response and measured and recorded RT to the nearest millisecond, starting at the onset of the pair of stimuli for simultaneous presentation and at the onset of the second stimulus for sequential presentation. The stimuli to be presented were displayed on a computer terminal next to the experimenter, who inserted the proper stimulus card(s) into the tachistoscope. After a trial had ended and while new stimulus materials were being inserted, a blank field with a fixation dot was always present. A trial lasted 6-7 sec.

A session lasted 50 to $60 \mathrm{~min}$, with each subject running ordinarily for only one session in a day, but occasionally for two sessions, with at least an hour between sessions. A session consisted of $\mathbf{4 8}$ practice trials, followed by 384 trials, half of which required a "same" response. Except for this constraint, each pair of stimuli occurred an equal number of times. Thus, when the rule for sameness was physical identity, each of the 4 "same" pairs was presented 48 times and each of the 12 different pairs was presented 16 times. When the rule for sameness involved one of the three dimensions, each of the 16 pairs was presented 24 times. The stimulus presentations were randomized by the computer in blocks of $\mathbf{4 8}$ so that each pair within each response type occurred equally often but there was no delay between successive blocks. Thus, from the point of view of the experimenter and the subject, a continuous session of 384 trials was run. A subject was allowed to take a short break during the session if desired.

A median RT was computed by the computer for all correct trials in a session for each of the 16 pairs of stimuli. These 16 median scores for each subject were the basis of all further analyses.

For each set of stimuli, four different rules for sameness were used, one for physical identity and one for each of the three dimensions. A single rule for sameness was used for a single session in order to minimize confusion about the rule being used. In both experiments, each subject used each of the rules for sameness. At the beginning of the experiment, each subject was informed of all the conditions and sameness rules that would be used and was instructed to "respond as rapidly as you can while taking care not to make errors." At the beginning of each session, the subject was instructed about the sameness rule to be used in that session.

\section{Subjects}

All subjects were Yale undergraduates, graduate students, or staff members in the Department of Psychology. All were paid 
to participate. In the first experiment, 16 subjects were used, each in eight different sessions. In the second experiment, 8 subjects were used, each in four different sessions. All except 1 subject had been used previously in experiments of this type; the one new subject was given additional practice.

\section{EXPERIMENT 1A: SIMULTANEOUS PRESENTATION, 6, 10, VI, $X$}

\section{Method}

Experimental design. Experiment 1 used both a simultaneous and a sequential presentation, and, although for purposes of analysis and exposition it is preferable to treat these two conditions separately, the two conditions were fully integrated into a single experimental design.

There were four tasks, differing only in the sameness rule used. These four tasks were arranged in a Latin square with four subjects and four sessions, and within this Latin square no sameness rule followed another more than once. This $4 \times 4$ Latin square was expanded to an $8 \times 8$ square by alternating the simultaneous and sequential presentations for each task, with one subject always having simultaneous before sequential and another subject having the reverse order, for each sequence of sessions used in the $4 \times 4$ Latin square. This arrangement meant that, although the basic design was an $8 \times 8$ Latin square, when only data for simultaneous or sequential presentation were used, a $4 \times 4$ Latin square existed, with two subjects for each sequence of sessions.

Two groups of eight subjects were used with the same Latin square arrangement. The groups differed in amount of experience in experiments of this type, and each group had a different experimenter. However, the factors of experience and experimenter did not affect the results, so for all analyses the data for all 16 subjects were pooled.

Stimuli and Tasks. The four stimuli used were the Arabic and the Roman numerals $6,10, \mathrm{VI}$, and X. The exact stimuli used are shown in Figure 1, in two of the lower left and upper right arrangements used. The characters were Letraset 725 , Helvetica Medium. The line thickness was $1.0 \mathrm{~mm}$, and the height of each numeral was $8.0 \mathrm{~mm}$ (visual angle of $.36 \mathrm{deg}$ ). The widths of the $6,0, V$, and $X$ were $6.0 \mathrm{~mm}$. The bottoms of the top numerals and the tops of the bottom numerals were at the horizontal midline. When a numeral contained a single digit, it was centered $8 \mathrm{~mm}$ to the right or left of fixation. When the numeral contained two digits, the digit nearer the fixation point was centered in the same way as the single digits and the second digit was centered $16 \mathrm{~mm}$ from fixation. Even though a fixation dot was always present between trials, the subjects were not asked to maintain fixation. Its purpose was simply to provide general orientation.

The stimuli vary orthogonally in three dimensions: Length (long or short), System (Arabic or Roman), and Name (six or ten). These three dimensions differ along the continuum from physical to cognitive: Length is a clearly physical dimension, one which needs

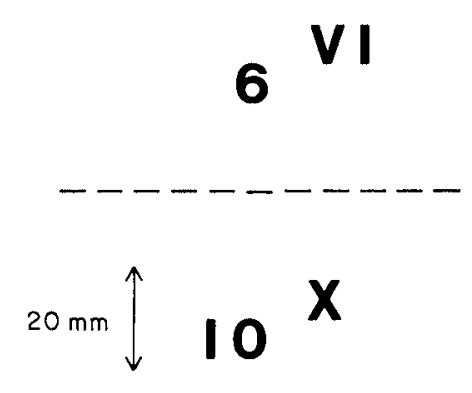

Figure 1. The four stimuli used in Experiment 1, in 2 of the 16 pair configurations used with simultaneous presentation. no semantic label, even though one might be used. System is a more arbitrarily defined dimension of the stimuli, yet this cognitive dimension can be changed into a reasonably physical one, since the Roman numerals have nothing but straight lines while both of the Arabic numerals have curved lines. Name as a dimension is certainly cognitive, however, since the stimuli 10 and $X$ and 6 and VI have little in common other than their arbitrarily assigned names. Thus, these three dimensions provide us with the opportunity to clarify the role of physical and cognitive dimensions in stimulus comparison.

The 16 pairs of these four stimuli form four groups with regard to the three dimensions defining the stimuli: Physically Identical $(6$ 6, 10 10, VI VI, X X); Same Length, different System and Name $(6 \times, X 6$, VI 10, 10 VI); Same System, different Length and Name (6 10, 10 6, VI X, X VI); and Same Name, different Length and System (6 VI, VI 6, 10 X, X 10).

These stimulus groups will be referred to by the dimension on which the stimulus pairs are the same, but it should be remembered that the stimuli always differ on the two dimensions on which they are not the same.

Four tasks were used, involving the four possible sameness rules: Physical, the "same" response being required only for the four Physically Identical pairs; Length, requiring the "same" response for the Physically Identical pairs plus the four Same Length pairs; System, requiring the "same" response to Physically Identical pairs plus the Same System pairs; and Name, requiring the "same" response for Physically Identical pairs plus the Same Name pairs. Except for the Physical rule for sameness, the other three rules require a response of "same" for Physically Identical pairs plus one of the other three groups of pairs, so all factors concerning number and types of stimuli requiring the "same" response are completely balanced.

\section{Results and Discussion}

Statistical analyses. Several analyses of variance that involved all four sameness rules were performed, and for these analyses the Latin-square design was used, with a residual error term that did not include order effects. For specific comparisons between sameness rules, $t$ tests based on the Latin-square residual error term were used. For comparisons between responses or stimulus pairs within a given sameness rule, individual $t$ tests were used because, within a rule, there was no confounding with order. Frequently, however, simple binomial sign tests were sufficient to establish the reliability of a result. Unless otherwise specified, significance levels of .01 were used. However, many of the important results come from an overall pattern of data rather than from the metric and nonmetric analyses, so the specific tests of significance, while usually available, are not as important as the overall pattern.

Session effects were small compared with other effects. For "same" responses, there was a decrease of $36 \mathrm{msec}$ from the first to the third of the four sessions that involved simultaneous presentation, the third session being fastest. For "different" responses, there was a nonsignificant decrease of $19 \mathrm{msec}$ from the first to the third session. There was no evidence of systematic differential effects of session on the experimental variables of interest, so the only function of these sessions effects is to provide a more sensitive statistical test by their inclusion in the analysis of variance. 
Table 1

Simultaneous Presentation, Stimuli 6, 10, VI, X: Mean RT (in Milliseconds) and Mean Errors per Trial for "Same" and "Different" Responses and Four Sameness Rules

\begin{tabular}{|c|c|c|c|c|c|c|c|c|c|c|}
\hline \multirow[b]{3}{*}{ Response } & \multicolumn{10}{|c|}{ Sameness Rule } \\
\hline & \multicolumn{2}{|c|}{ Physical } & \multicolumn{2}{|c|}{ Length } & \multicolumn{2}{|c|}{ System } & \multicolumn{2}{|c|}{ Name } & \multicolumn{2}{|c|}{ Mean } \\
\hline & $\mathrm{RT}$ & Errors & $\mathrm{RT}$ & Errors & RT & Errors & RT & Errors & $\mathrm{RT}$ & Errors \\
\hline $\begin{array}{l}\text { "Same" } \\
\text { "Different" }\end{array}$ & $\begin{array}{l}400 \\
437\end{array}$ & $\begin{array}{l}.024 \\
.024\end{array}$ & $\begin{array}{l}446 \\
480\end{array}$ & $\begin{array}{l}.038 \\
.038\end{array}$ & $\begin{array}{l}484 \\
537\end{array}$ & $\begin{array}{l}.045 \\
.036\end{array}$ & $\begin{array}{l}523 \\
609\end{array}$ & $\begin{array}{l}.046 \\
.037\end{array}$ & $\begin{array}{l}463 \\
516\end{array}$ & $\begin{array}{l}.038 \\
.034\end{array}$ \\
\hline Mean & 419 & .024 & 463 & .038 & 510 & .040 & 566 & .041 & 490 & .036 \\
\hline
\end{tabular}

Overall results. The data in Table 1 show the mean RTs and errors for "same" and "different" responses for each of the sameness rules. Mean "same" responses were $53 \mathrm{msec}$ faster than mean "different" responses, a result in line with a considerable literature showing the same result (see Nickerson, 1978, and Proctor, 1981). The four rules for sameness gave RTs increasing for the four tasks, a sameness rule of Physical Identity being fastest, Length next fastest, System next, and Name being the slowest. The range of mean RTs from fastest to slowest was $147 \mathrm{msec}$, and all differences between rules were statistically significant, both for overall means and for each type of response separately. In addition, there was a significant interaction between type of response and sameness rule, with System and Name rules showing a greater difference between the two types of response.

Mean errors were, on the whole, quite small, and in general reflected the results for RT data with regard to sameness rule, but showed little difference between the two types of response.

Taken as a whole, these results indicate that processing is easiest for the most physical dimension

Table 2

Simultaneous Presentation, Stimuli 6, 10, VI, X: Mean RT (in Milliseconds) for Each Stimulus Pair and Each Sameness Rule

\begin{tabular}{|c|c|c|c|c|}
\hline \multirow{2}{*}{$\begin{array}{l}\text { Stimulus } \\
\text { Pair }\end{array}$} & \multicolumn{4}{|c|}{ Sameness Rule } \\
\hline & Physical & Length & System & Name \\
\hline & \multicolumn{4}{|c|}{ Physical Identity } \\
\hline 66 & $390^{*}$ & $410^{*}$ & $411^{*}$ & $439 *$ \\
\hline 1010 & $389^{*}$ & $410^{*}$ & $418^{*}$ & $436^{*}$ \\
\hline VI VI & $429 *$ & $446^{*}$ & $449 *$ & $477^{*}$ \\
\hline \multirow[t]{2}{*}{$X X$} & $394^{*}$ & $410^{*}$ & $426^{*}$ & $444^{*}$ \\
\hline & \multicolumn{4}{|c|}{ Same Length } \\
\hline $6 \mathrm{X}$ & 425 & $460^{*}$ & 509 & 610 \\
\hline \multirow[t]{2}{*}{$10 \mathrm{VI}$} & 456 & $486^{*}$ & 569 & 630 \\
\hline & \multicolumn{4}{|c|}{ Same System } \\
\hline \multirow{3}{*}{$\begin{array}{l}610 \\
\text { VI X }\end{array}$} & 440 & 483 & $510^{*}$ & 567 \\
\hline & 460 & 489 & $573^{*}$ & 631 \\
\hline & \multicolumn{4}{|c|}{ Same Name } \\
\hline $6 \mathrm{VI}$ & 412 & 467 & 537 & $617^{*}$ \\
\hline $10 X$ & 426 & 480 & 532 & $577 *$ \\
\hline
\end{tabular}

(Length) and most difficult for the most cognitive dimension (Name), with the dimension intermediate between a physical and cognitive dimension (System) also being intermediate in processing speed. This overall picture, while simple, does not clarify the ways in which the processing of the different types of dimensions differ, or even whether cognitive dimensions are processed as dimensions in the same way that physical dimensions are. To elucidate these matters requires examination of more detailed data.

Physically Identical pairs. Table 2 shows mean RTs for each of the 10 stimulus pairs for each of the sameness rules, with data collapsed across order for the stimulus pairs that are different. Of the Physically Identical pairs, within each sameness rule (and therefore, on the average) the stimulus VI is responded to as "same" more slowly than the other three stimuli, with an average difference of $35 \mathrm{msec}$. This difference is statistically significant, but no other differences between identical pairs are. This slower responding of "same" to the VI is similar to that found by Podgorny and Garner (1979) in a same-different experiment using uppercase letters. Their results showed that the "same" response was made more slowly to letters $M, N, V$, and $W$, which have one or more diagonal lines and bear an obvious similarity to the VI stimulus of the present experiment.

The effect of the sameness rule on speed of processing identical pairs is the same as that overall, with difficulty of processing increasing from the Physical Identity rule to the Name rule. This finding of an increase in RT to physically identical stimuli as the sameness rule is changed is quite in line with many other results (see Posner, 1978). Some of the adjacent pairs are significantly different at only the .05 level (Length vs. Physical and Name vs. System), and the difference between System and Length is not statistically significant. Nevertheless, because the ordering of the different rules is exactly the same as the overall ordering and is the same ordering for each of the four identical stimulus pairs, it seems reasonable to assume that the difficulty of processing identical pairs is determined by the overall difficulty of using each of the dimensions as the basis for a sameness rule. 
However, although the ordering of difficulty of the identical pairs for different sameness rules is the same as the ordering of overall results, the magnitudes of the effects are considerably less for identical stimulus pairs than for "same" responses overall, which include nonidentical stimulus pairs for which the "same" response is required. The contrast is even greater if RTs to physically identical stimulus pairs are directly contrasted with "same" RTs for the nonidentical stimulus pairs. For physically identical pairs, the mean RTs for the four sameness rules are successively $400,419,426$, and $449 \mathrm{msec}$. The mean "same" RTs for the latter three sameness rules for only those stimulus pairs that are nonidentical are 473, 542, and 597 msec. Thus, the "same" data shown in Table 1 are a composite of two sets of stimulus pairs that give quite different results across sameness rules, with identical stimuli being only little affected by the sameness rule and the nonidentical stimulus pairs being greatly affected. This combination of two distinctly different functions results in the overall analysis's showing a large and statistically significant effect of response type and an interaction between sameness rule and response type.

If an analysis is performed only with nonidentical stimulus pairs, excluding physically identical stimulus pairs and thus also all data for the Physical Identity sameness rule, the mean RT for "same" responses is $537 \mathrm{msec}$ and that for "different" responses is $543 \mathrm{msec}$. This difference between types of response is not statistically significant, nor is the interaction between response type and sameness rule. Thus, the overall result showing such results is somewhat misleading, and, while there may be a faster "same" than "different" response, its magnitude is to a large extent due to the very fast processing of physically same stimulus pairs.

Task difficulty and dimensional discriminability. There is a clear ordering of difficulty for the four sameness rules, even though, except for the Physical Identity rule, there is no difference in the number of stimuli that satisfy the definition of sameness. The next question is: What accounts for the ordering of difficulty of the sameness rules? One possible answer is simply that the three dimensions differ in discriminability, and these differences determine processing difficulty.

In order to address this question, use can be made of data for the six pairs of different stimuli with the Physical Identity rule, because each of these pairs requires the "different" response; therefore, the RTs for these six pairs of different stimuli with this sameness rule should reflect the discriminabilities of the stimuli and of the dimensions from which they were generated. A two-dimensional scaling solution for these four stimuli based on the RTs for the "different" responses was carried out, and the results are shown in Figure 2. For this solution, each

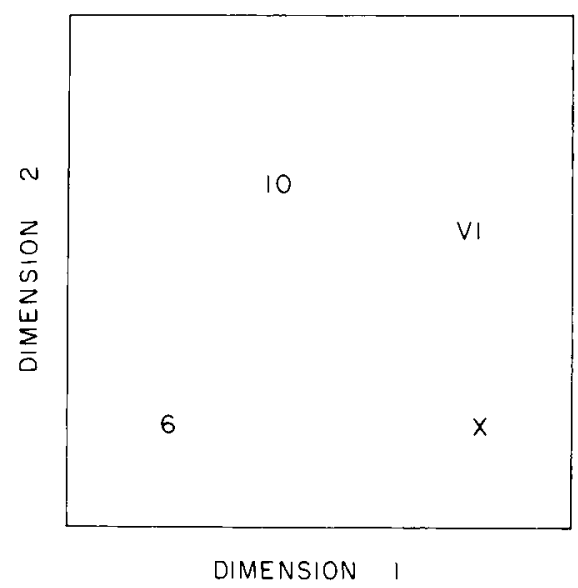

Figure 2. Stimuli 6, 10, VI, X: A two-dimensional euclidean scaling solution for these stimuli based on RTs for "different" responses with the Physical Identity rule.

RT for a pair of stimuli was subtracted from a constant $(513 \mathrm{msec})$ whose value was determined to give an optimum solution. The resultant numbers were then assumed to be a measure of discriminability, with high numbers (originally low RTs) indicating high discriminability. The scaling, which was carried out graphically, with a euclidean metric, gave a solution in which each stimulus could be placed in two dimensions within a 2-msec accuracy.

The solution leads to the four stimuli's being arranged in a roughly trapezoidal shape, with two dimensions which are easily identified as Length and System. Discriminability is, on average, greater for System than for Length, as of course the original RT data themselves indicate. The Same Length pairs differ in Name and System and give faster RTs than the Same System pairs, which differ in Name and Length. Thus, System is more discriminable than Length. Furthermore, RTs for Same Name stimulus pairs are fastest because such pairs differ in both System and Length redundantly. There is therefore an ordering of the discriminabilities such that Same Name stimuli are most discriminable, Same System stimuli are next most discriminable, and Same Length stimuli are the least discriminable, an ordering which exectly reflects the difficulty of the sameness rules.

The scaling solution in Figure 2 so obviously reflects the two most physical dimensions of Length and System that it is questionable that Name is operating as a dimension at all, at least with the Physical Identity rule for sameness. The pairs of stimuli that are Same Name lie on the diagonals of the trapezoid, and it is as easy to describe the Same Name stimuli as the biconditional of the dimensions of Length and System as it is to assume that Name is a dimension in the same sense that the others are. In other words, the ordering of difficulty of the three sameness rules 
involving a dimension would have been the same if two dimensions plus the biconditional rule had been used for the sameness rules.

Discriminability or ease of processing? Despite the easy interpretation that the difficulty of using a sameness rule is simply related to the discriminability of the dimension on which the rule is based, further internal evidence suggests that, at least to some extent, the different stimulus pairs provide different ease of processing and that the different sameness rules change the mode of processing of the stimuli, probably in an interactive way.

The strongest evidence for this assumption is based on the single most apparent property of the data shown in Table 2: Every one of the 10 rows representing different stimulus pairs increases in exactly the same order across the four columns representing the different rules for sameness, and the statistical probability of this event is very low indeed. The importance of this relation in understanding how these stimuli are processed is that this consistent ordering occurs even though, for pairs of physically nonidentical stimuli, the different sameness rules require a change from a "same" to a "different" response or vice versa. Thus, the stimulus pair 610 increases regularly across sameness rules even though this stimulus pair requires a response of "same" for the system rule but a response of "different" for the other three rules.

The failure of the requirement of a "same" rather than a "different" response to be sufficiently strong to prevent the consistent ordering of difficulty across columns suggests, but does not prove, that the response is less important than is the actual pair being processed. Further and stronger evidence that ease of processing may be more important than the response comes from comparisons between stimulus pairs within the same group. For example, the pairs $6 \mathrm{X}$ and $10 \mathrm{VI}$ are Same Length, but $6 \mathrm{X}$ is responded to faster with each sameness rule. If this difference in speed of processing is due to discriminability, then the relation between these two pairs should have reversed when a "same" response was required, since lack of discriminability should produce a fast "same" response but a slow "different" response. A similar strong pattern exists with Same System stimuli, in which the pair 610 is always processed faster than the pair VI X, regardless of the nature of the response or the rule for sameness. In fact, it is very easy to summarize these results for Same Length and Same System stimulus pairs as follows: Short stimuli are easier to process than long stimuli, and Arabic numerals are easier to process than Roman numerals. When the results are described this way, it is easy to see why the VI was consistently high in RT for pairs of stimuli that were physically identical, since the VI is neither short nor Arabic, both or either of which are required for fast processing.
Pairs of stimuli having the Same Name do not show a consistent pattern across sameness rules: For the Physical Identity rule, as can be seen from Table 2 or from Figure 2, the stimulus pair $6 \mathrm{VI}$ is more discriminable and therefore processed faster than the pair $10 \mathrm{X}$. This same relation holds when the sameness rule is Length, and with both rules the difference is statistically significant at the .05 level. Thus, stimuli with the name "six" are processed faster than those with the name "ten." With the sameness rule of System, processing of the 6 VI stimulus pair is nonsignificantly slower, but with the sameness rule of Name, processing of the 6 VI pair is significantly slower than the $10 \mathrm{X}$ pair.

This set of relations suggests that when the sameness rule is Physical Identity or the physical dimension of Length, the discriminability relations shown in Figure 2 are primary in determining how the stimuli are processed. When the clearly cognitive sameness rule of Name is used, processing changes so that these discriminability properties are less important than the name properties themselves. With the dimension that has both physical and cognitive properties, System, the processing shows a compromise between these conflicting properties. For Same Length and Same System stimulus pairs, ease of processing remains the same for all sameness rules, and it is not illogical that highly discriminable stimuli are also easy to process, at least when a complex sameness decision is required. When, however, the discriminability of the stimuli is in conflict with a processing requirement, as with the Name rule, then the sameness rule takes priority over the discriminability property in determining how processing is to be done.

Error data are not shown in Table 2 because too few errors were made to allow meaningful detailed analysis. There is one aspect of the error data that supports the idea that the nature of the processing changes with different sameness rules. From Table 1 it can be seen that errors with "same" responses increase from the Physical through the Name rule. However, the errors made to Physically Identical stimuli actually decrease when a rule for sameness is used other than Physical, from the .024 obtained for the Physical Identity rule to, successively, .012, $.008, .006$. While the number of errors involved is too few $(18,12$, and 9 , respectively) to make meaningful statistical comparisons between the three dimensional rules, the average from these three rules is significantly lower than the mean for the Physical Identity rule, this difference occurring for 14 of 15 subjects, the 16th subject having made no errors for these stimuli. This lowered error rate, of course, is accompanied by a substantially increased $\mathrm{RT}$ to these stimuli, as seen in Table 2 . In order for average errors for "same" responses to increase while those to Physically Identical stimuli pairs decrease, clearly the error rate for those stimulus pairs that are the same 
by the dimensional rule but not by the physicallysame rule had to increase markedly.

These results, taken together, show that the speed of processing these stimulus pairs is not simply a matter of dimensional discriminability, but that the nature of the processing changes with changes in the sameness rule and that different pairs of stimuli are processed more or less easily, some for all sameness rules, while other pairs have an altered ease of processing, depending on the sameness rule. Thus, even though the confounding factor of an increase in the number of stimuli that satisfy the sameness rule with changes in the rule has been eliminated, it is clear that there are meaningful differences in both ease and nature of processing with different sameness rules. These differences and their nature are sufficiently strong to suggest that a cognitive dimension such as Name is not processed as a dimension in the same way at all as the more physical dimensions are.

Stimulus order effects. The data for all pairs of different stimuli are shown in Table 2 without regard to the left-right order of the stimuli because there were no consistent effects of order. The mean difference between the orders for the 24 stimulus pairs (i.e., 6 pairs in each of four instructions) was $8 \mathrm{msec}$, with a standard deviation of $6 \mathrm{msec}$ and a maximum difference of $23 \mathrm{msec}$. These data are mentioned because there are important order effects with sequential presentation, and these are, in effect, control data for that condition.

\section{Summary and Conclusions}

(1) Different rules for sameness lead to different speeds of processing, and these differences are not a function of differences in number of stimuli that satisfy the definition of sameness. For the three dimensions used in this experiment, processing was fastest for the clearly physical dimension, slowest for the clearly cognitive dimension, and intermediate for the dimension that was both physical and cognitive. All three dimensional rules, however, gave slower processing than a sameness rule of Physical Identity.

(2) The ease of processing the three dimensions is also correlated with the discriminability of the dimensions as determined from the RTs to different stimuli with a sameness rule of Physical Identity.

(3) Despite that correlation, it is clear that some stimulus pairs (and probably individual stimuli) simply are processed more easily than others, regardless of whether the processing leads to a response of "same" or of "different." In particular, short stimuli are processed faster than long stimuli, and Arabic numerals faster than Roman numerals.

(4) Different sameness rules also lead to different types of processing, including some changes in how particular stimulus pairs are processed.

(5) Although the structure of the stimulus set is dimensionally defined, it is not clear that all such imputed dimensions function in the same way. There is a special question as to whether the cognitive dimension of Name functions as a dimension for which discriminability is a meaningful property, as it is for the physical dimension of Length.

The clarification of some of these issues, especially the processing role of the cognitive dimension of Name, was the purpose of Experiment 1B, which used the same stimuli but sequential presentation.

\section{EXPERIMENT 1B: SEQUENTIAL PRESENTATION, 6, 10, VI, $\mathrm{X}$}

\section{Method}

As indicated under Experiment 1A, these two experiments were run as a single integrated experiment, with the same subjects and with the simultaneous and sequential presentations for each subject alternated. The only difference was that, for Experiment 1B, the left-hand stimulus came on for $500 \mathrm{msec}$ before the right-hand stimulus.

\section{Results and Discussion}

The same basic statistical procedures used in Experiment $1 \mathrm{~A}$ were used here. Session effects were statistically significant for both "same" and "different" responses, with a decrease of $56 \mathrm{msec}$ from the first to the third of the four sequential sessions in each case. These session effects were about twice as great as those obtained with simultaneous presentation, and although that fact might have been of some interest in its own right, for the purposes at hand, its main effect was once again to provide a more sensitive statistical test by eliminating the confounding effect of session from the error variance in the analysis of variance. There appeared to be no obvious ways in which differences between experimental factors were influenced by the session effects.

Since most of the results for sequential presentation are equivalent to those for simultaneous presentation, they will be presented more briefly. The major differences involved the use of the Name rule for sameness, and they will be emphasized.

Overall results. The data in Table 3 show overall mean RTs and errors for both response types and each sameness rule. The average RT was $385 \mathrm{msec}$, which is $105 \mathrm{msec}$ faster than that obtained with simultaneous presentation, and presumably this greater speed primarily reflects a readiness factor that is relevant when one of the two stimuli is presented in advance. Mean "same" responses were $48 \mathrm{msec}$ faster than mean "different" responses, a difference similar to that obtained with simultaneous presentation. The four rules for sameness gave RTs increasing from Physical Identity to System, but Name was not significantly different from System. There was in addition a significant interaction between type of response and sameness rule, although the pattern of the interaction is less clear than that 
Table 3

Sequential Presentation, Stimuli 6, 10, VI, X: Mean RT (in Milliseconds) and Mean Errors per Trial for "Same" and "Different" Responses and Four Sameness Rules

\begin{tabular}{|c|c|c|c|c|c|c|c|c|c|c|}
\hline \multirow[b]{3}{*}{ Response } & \multicolumn{10}{|c|}{ Sameness Rule } \\
\hline & \multicolumn{2}{|c|}{ Physical } & \multicolumn{2}{|c|}{ Length } & \multicolumn{2}{|c|}{ System } & \multicolumn{2}{|c|}{ Name } & \multicolumn{2}{|c|}{ Mean } \\
\hline & RT & Errors & RT & Errors & RT & Errors & RT & Errors & RT & Errors \\
\hline "Same" & 316 & .025 & 357 & .030 & 388 & .032 & 383 & .036 & 361 & .031 \\
\hline "Different" & 366 & .024 & 396 & .032 & 430 & .029 & 442 & .023 & 409 & .027 \\
\hline Mean & 341 & .025 & 377 & .031 & 409 & .030 & 412 & .029 & 385 & .029 \\
\hline
\end{tabular}

obtained with simultaneous presentation. Error data again basically reflect the RT data.

Overall, the major difference between simultaneous and sequential presentation is that Name ceases to be such a very poor dimension to use as a sameness rule and, with sequential presentation, is processed as fast as System.

Physically Identical pairs. Table 4 shows mean RTs for each of the stimulus pairs for each of the sameness rules, with data collapsed across order for the stimulus pairs that are different. On the average and for each sameness rule, the stimulus VI is responded to more slowly than are the other three stimuli, replicating the result with simultaneous presentation.

The effect of the sameness rule on RT is the same as that overall, with there being a regular progression from the rules for Physical Identity to Length to System, but with no real differences between the System and the Name rules. Name and System are not significantly different from each other, but both are significantly slower than Physical Identity. Other differences hover around a significance level of .05 ,

Table 4

Sequential Presentation, Stimuli 6, 10, VI, X: Mean RT (in Milliseconds) for Each Stimulus Pair and Each Sameness Rule

\begin{tabular}{|c|c|c|c|c|}
\hline \multirow{2}{*}{$\begin{array}{c}\text { Stimulus } \\
\text { Pair }\end{array}$} & \multicolumn{4}{|c|}{ Sameness Rule } \\
\hline & Physical & Length & System & Name \\
\hline & \multicolumn{4}{|c|}{ Physical Identity } \\
\hline $\begin{array}{l}66 \\
1010 \\
\text { VI VI } \\
\times X\end{array}$ & $\begin{array}{l}310^{*} \\
312^{*} \\
333^{*} \\
310^{*}\end{array}$ & $\begin{array}{l}326^{*} \\
333^{*} \\
342^{*} \\
334^{*}\end{array}$ & $\begin{array}{l}342^{*} \\
341^{*} \\
368^{*} \\
359^{*}\end{array}$ & $\begin{array}{l}349^{*} \\
344^{*} \\
377^{*} \\
352^{*}\end{array}$ \\
\hline $\begin{array}{l}6 \mathrm{X} \\
10 \mathrm{VI}\end{array}$ & $\begin{array}{l}361 \\
367\end{array}$ & $\begin{array}{l}\text { Sam } \\
379^{*} \\
384^{*}\end{array}$ & $\begin{array}{l}\text { ngth } \\
425 \\
440\end{array}$ & $\begin{array}{l}442 \\
453\end{array}$ \\
\hline \multirow[t]{2}{*}{$\begin{array}{l}610 \\
\text { VIX }\end{array}$} & $\begin{array}{l}365 \\
380\end{array}$ & $\begin{array}{l}\text { Sam } \\
394 \\
402\end{array}$ & $\begin{array}{l}\text { stem } \\
408^{*} \\
438^{*}\end{array}$ & $\begin{array}{l}421 \\
451\end{array}$ \\
\hline & \multicolumn{4}{|c|}{ Same Name } \\
\hline $\begin{array}{l}6 \mathrm{VI} \\
10 \mathrm{X}\end{array}$ & $\begin{array}{l}361 \\
366\end{array}$ & $\begin{array}{l}389 \\
399\end{array}$ & $\begin{array}{l}429 \\
426\end{array}$ & $\begin{array}{l}429 * \\
392^{*}\end{array}$ \\
\hline
\end{tabular}

*"Same" responses. but because of the fact that this ordering is the same as the overall ordering and the same as that found with simultaneous presentation, it is reasonable to assume that the order of rule difficulty is the same as that in Table 3 for overall data.

Again, the magnitudes of the effects of sameness rule are small for Physically Identical stimuli as contrasted with the effect of these rules on "same" responses for nonidentical stimulus pairs. Thus, the "same" data in Table 3 are a composite of two sets of stimulus pairs that give quite different results across sameness rules. In an analysis that uses only physically nonidentical stimulus pairs, the mean RT for "same" is $405 \mathrm{msec}$ and that for "different" is $423 \mathrm{msec}$. While this difference is still statistically significant, it is greatly reduced from that obtained when Physically Identical stimuli are included in the calculation. There is also a statistically significant interaction between type of response and sameness rule, an effect due to the fact that the difference between RTs for "same" and "different" responses is greater with the Name rule for sameness than with the other two rules.

Dimensional discriminability. With sequential presentation, there is no difference between the use of the System and the Name rules for sameness. Is this contrast with the result found with simultaneous presentation due to (or reflected by) a change in the picture of dimensional discriminability? There is no evidence that it is: the overall correlation between the six pairs of different stimuli with the Physical Identity rule for sameness in the simultaneous and sequential presentations is .80 , a high correlation despite the smaller range of numbers with sequential presentation. Thus, the two-dimensional scaling solution of discriminabilities shown in Figure 2 adequately represents the discriminabilities with sequential presentation. At a more detailed level, within each type of nonidentical stimulus pair in Table 4 (Physical Identity rule), one pair gives shorter RTs than the other, and these pairs all have the same relation as that in Table 2 . Still further, the average RTs for the three types of nonidentical pairs show the same rank order as they did with simultaneous presentation, although the differences in RT are much smaller. 
Thus, to summarize this point, the improved performance with the Name rule for sameness with sequential presentation is not due to a change in the pattern of discriminabilities between the two types of presentation. The improvement must, therefore, be some function of the way that the Name dimension is used in processing.

Ease of processing. The general picture showing that the particular stimulus pairs, interacting with the sameness rule, provide differences in ease of processing is very similar to that found with simultaneous presentation, with the exception that the Name rule shows easier processing. All 10 stimulus pairs produce an orderly increase across the four sameness rules, with three exceptions, all involving faster processing for Name rule than for System rule: With pairs X X, $6 \mathrm{VI}$, and $10 \mathrm{X}$, the RTs with Name are all equal to or faster than the RTs with System. In fact, the "same" response is made, on the average, faster with nonidentical pairs using the Name rule than with nonidentical pairs using the System rule. Thus, there clearly is an improved speed of processing of the Name dimension with sequential presentation.

In examining particular stimulus pairs, the same advantage within each type of sameness rule holds, as with simultaneous presentation, regardless of the response required: Pairs of short stimuli are processed faster than pairs of long stimuli for Same Length pairs for all sameness rules; Arabic pairs are processed faster than Roman pairs for Same System pairs for all sameness rules; and the $6 \mathrm{VI}$ pair is processed faster than the $10 \mathrm{X}$ pair for the Physical Identity and Length rules, but this relation reverses for the System rule and especially for the Name rule. Once again, there is little evidence that dimensional discriminability is a major factor in how these pairs of stimuli are processed, especially for the System and Name rules for sameness. It may be a factor when the Physical Identity or Length rule is used.

Error data again support the idea that the nature of processing changes with different sameness rules. With Physically Identical stimuli, the mean error for Length, System, and Name rules for sameness was .015 , compared with a mean error of .025 for the Physical Identity rule, and this difference was displayed by 14 of the 15 subjects who made any errors. Thus, subjects adjust their processing when a rule other than Physical Identity is used, with fewer errors for physically identical stimuli than for nonidentical stimuli requiring the "same" response.

Stimulus order effects with Name rule. With sequential presentation, a major result is that processing speed for the Name rule is improved relative to the other sameness rules. The evidence concerning why the improvement occurs lies in stimulus order effects. Such effects were inconsequential with simultaneous presentation but were very strong with sequential presentation and the Name rule for same- ness. The stimulus pairs $6 \mathrm{VI}$ and $10 \mathrm{X}$ require the "same" response with the Name rule. The mean RT for these two pairs in the order given (Roman numeral second) is $438 \mathrm{msec}$. In the reverse order (Arabic numeral second), mean RT is $383 \mathrm{msec}$, a highly significant difference of $55 \mathrm{msec}$. (The difference is also significant for each pair considered alone.) In fact, this latter mean RT is substantially less than that for "same" responses to nonidentical stimuli with the System rule and is approximately the same as that for "same" responses to nonidentical stimuli with the Length rule.

These order effects with the Name rule are not restricted to stimuli requiring the "same" response. Stimulus pairs having the same length differ also in whether the first or second stimulus is Arabic or Roman, but these pairs require the "different" response with the Name rule for sameness. The mean RT for the pairs $6 \mathrm{X}$ and $10 \mathrm{VI}$ (Roman numeral second) is $466 \mathrm{msec}$, while for these same pairs in reverse order (Arabic numeral second), the mean RT is $428 \mathrm{msec}$, a significant difference of $38 \mathrm{msec}$. The consistency of these order effects can be seen by noting that all 16 subjects showed the effect with the "same" response and 15 subjects showed it with the "different" response.

The order effect, even with the Name rule, occurs only when the two stimuli differ in System. The Same System pairs, 610 and VI X, require a "different" response with the Name rule, and for these stimuli the order effect is $2 \mathrm{msec}$ for the Arabic pair and $6 \mathrm{msec}$ for the Roman pair.

Still further, the order effect occurs only with the Name rule for sameness. There are four pairs of stimuli that differ in System, each used with both the Length and System rules for sameness. Of these eight opportunities for a faster RT with the Arabic numeral second, only two showed a difference in that direction, and the mean order effect was $6 \mathrm{msec}$ in favor of the Roman numeral second.

Results with simultaneous presentation show Arabic numerals to be processed faster than Roman numerals, so the order effect found with sequential presentation might be little more than a reflection of the greater ease of processing Arabic numerals. However, with simultaneous presentation, short numerals were also processed faster than long numerals, yet no such order effects occurred with stimuli differing in Length. There are four pairs of stimuli that differ in Length, each used with both Length and System rules for sameness. Of these eight opportunities for a faster RT with the short stimulus second, five occurred in that direction, and the mean difference was less than $2 \mathrm{msec}$ in the expected direction. Thus, the large order effect requires stimuli both differing in System and having the Name rule for Sameness.

Name as a processing mechanism. The present data do not provide an unequivocal answer to the 
question of which processing construct is appropriate to an explanation of the order effects for the Name rule and, in turn, why the Name rule for sameness is so much more effective with sequential presentation than with simultaneous presentation. There are at least three possibilities.

One possibility is that the match is done on the basis of name codes, and that it simply takes longer to retrieve a name code from the unfamiliar Roman numerals than from the familiar Arabic numerals. Certainly the evidence from simultaneous presentation suggests this possibility, since pairs of Arabic numerals were processed faster than pairs of Roman numerals for all sameness rules. However, pairs of short numerals were also processed faster than pairs of long numerals, again for all sameness rules and with simultaneous presentation, yet no stimulus sequence effect for differences in length occurred with sequential presentation. It is somewhat awkward to justify this possibility on the basis of evidence from simultaneous presentation when equivalent evidence for the length dimension shows no equivalent effect with sequential presentation.

A second possibility is that the name generated by the first stimulus provides differential activation for the name of the second stimulus, Arabic numerals being more easily activated than Roman numerals. Although this possibility is not clearly demonstrated by results of the present experiment, it is not at all refuted.

A third possibility is that the name code is used to generate a physical representation, and that the match is then made, not on the basis of Name, but on the basis of the generated figure. That such generation can occur has been amply demonstrated by Posner (1978, pp. 49-56) and by Tversky (1969). In the present experiment, however, the evidence most clearly suggests that, with the Name rule, subjects prepare for the second stimulus by generating (or maintaining) the Arabic form of the numeral, the form that is easier to process. Then when the Arabic form occurs, RT is very fast. In fact, when the second numeral is Arabic, processing speed approaches that which occurs when both the first and the second numerals are Arabic and the same. If generation does occur, it is conceivable that, with longer delays, RT would be as low as when Physically Identical stimuli are used.

None of these three processing suggestions is clearly proved or refuted. Inherent in all of the suggestions, however, is that Name acts more as a processing mechanism than as a dimension such as the more physical dimensions of Length and System, even though its role in the structure of the stimulus set is the same. In fact, with discriminability scaling of data from simultaneous presentation, there is no evidence that Name operates as a dimension at all. Yet, with sequential presentation, it has a very important role, one providing recoding or even generation of physical images. On the other hand, there is no evidence that the physical dimension of Length, for which there are strong differences in speed of processing of short rather than long stimuli, or System, which has a clear physical counterpart, can provide the kind of processing mechanism that Name provides.

\section{Summary and Conclusions}

The data obtained with sequential presentation confirm most of the conclusions based on data obtained with simultaneous presentation in that relations between "same" and "different" responses and between different rules, and between pairs of stimuli differing in length or system, or both, are essentially the same. However, the special role of the Name dimension is clarified.

(1) With sequential stimulus presentation, the Name dimension, when used as the rule for sameness, allows processing as fast as that of the System dimension, especially when the "same" response is used with nonidentical stimuli.

(2) Much of this improvement occurs because, with Name as the sameness rule, processing is much faster when the second numeral is Arabic rather than Roman, with either "same" or "different" responses. This result suggests that Name operates as a processing mechanism rather than as a dimension.

(3) Even though internal evidence shows that short stimuli are processed faster than long stimuli, the dimension of Length does not provide an equivalent processing mechanism.

\section{EXPERIMENT 2: SIMULTANEOUS PRESENTATION, 3, 4, 6, 7}

One finding of Experiment 1, especially strong with simultaneous presentation, was that the ordering of difficulty of the three dimensions, when they were used as the rule for sameness, was perfectly correlated with the physical-to-cognitive continuum of the dimensions: the physical dimension of Length was easier than the cognitive dimension of Name, and the dimension of System was intermediate. From this finding, it is tempting to generalize that differences in RT in the comparison task reflect differences in levels of processing and that differences in levels of processing are themselves a function of the extent to which a dimension can be processed peripherally rather than centrally.

Even though, by the logic of the situation, physical properties must be processed to some degree before cognitive properties can be comprehended and thus processed, some models of processing do not require such a simple ordering of ultimate processing speed. In particular, Posner's $(1978$, p. 35ff.) concept of isolable systems allows for the possibility that a cog- 
nitive dimensions can be processed faster than at least some physical dimensions. It is not easy to demonstrate faster processing of a cognitive dimension, because of the usual confounding of number of alternatives that satisfy the definition of sameness with the degree to which a dimension is cognitive. The present methodology, however, which maintains equality of number of alternatives while allowing a comparison of three different dimensions, makes such a demonstration at least more feasible.

The purpose of Experiment 2 was specifically to determine whether an unequivocally cognitive dimension could be processed faster than more physical dimensions, and what the nature of such processing would be. For this purpose, the highly overlearned cognitive dimension of magnitude of Arabic numbers was used, along with two other dimensions.

\section{Method}

Experimental design. Only simultaneous presentation was used, and, thus, four different tasks were required for each of the four different sameness rules, one rule being used per session, as before. The four tasks were arranged into a Latin square, with no sameness rule following another more than once. The same Latin square was repeated with two groups of four subjects each.

Stimuli and Tasks. The four stimuli were the Arabic numerals $3,4,6$, and 7. The exact stimuli are shown in Figure 3, in two of the pair configurations actually used. The characters were Letraset 348 , Univers 55 . Line thickness averaged $1.0 \mathrm{~mm}$, the height of each numeral was $9.5 \mathrm{~mm}$ (visual angle of $.43 \mathrm{deg}$ ), and the width of each numeral was $7.5 \mathrm{~mm}$. The locations of the numerals on the viewing card were the same as in Experiment 1 and are displayed in Figure 3. These particular numerals were used because the 3 and the 6 consist entirely of curved lines and the 4 and the 7 entirely of straight lines.

These stimuli vary orthogonally in three dimensions: Curvilinearity (curved or straight), Oddness (odd or even), and Magnitude (high or low). Curvilinearity is clearly a physical dimension, and the other two are clearly cognitive, having no obvious physical characteristics to define their two classes. Of the two cognitive dimensions, however, Magnitude is far more familiar to the average adult than is Oddness.

The 16 pairs of these four stimuli form the following four groups with regard to the dimensions defining the stimuli: Physically Identical (3 3, 4 4, 6 6, 7 7); Same Magnitude, different Oddness and Curvilinearity $(34,43,67,76)$; Same Oddness, different Magnitude and Curvilinearity $(37,73,46,64)$; Same Curvilinearity, different Magnitude and Oddness (3 6, 6 3, 4 7, 7 4).

Four tasks were used, involving the four possible sameness

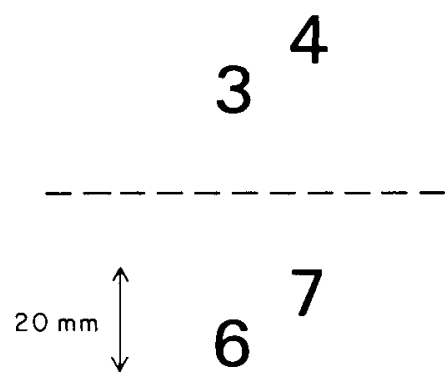

Figure 3. The four stimuli used in Experiment 2, in 2 of the 16 pair configurations used. rules: Physical, requiring a response of "same" only to Physically Identical pairs, and one rule for each of the dimensions, each rule requiring the "same" response to the Physically Identical pairs plus the appropriate additional group of stimulus pairs, as in Experiment 1 .

\section{Results and Discussion}

Statistical analyses. The statistical analyses were the same as those used for Experiment 1 . Because of the use of only eight subjects, however, a criterion of significance more lenient than the .01 level is more frequently necessary.

Session effects were significant, but at the .05 level. For "same" responses, there was a decrease in RT of $30 \mathrm{msec}$ from the first session to the fourth, while the analogous decrease was $35 \mathrm{msec}$ for "different" responses. As in Experiment 1, there was no evidence of any systematic effect of session on the experimental variables of interest, so the only function of the session effects is to provide a more sensitive statistical test by their inclusion in the analysis of variance.

Overall results. Table 5 shows overall mean RTs and errors for "same" and "different" responses for each of the sameness rules. Mean "same" responses were $39 \mathrm{msec}$ faster than mean "different" responses, a difference statistically significant. The four rules for sameness showed that the Physical Identity rule produced the lowest RTs, followed by Magnitude, Oddness, and Curvilinearity, in order, for both "same" and "different" responses. RTs for the Physical Identity rule were significantly faster than those for any of the dimensional rules, for both types of responses. Oddness and Curvilinearity rules did not differ significantly, but these two rules gave, on the average, slower RTs than did the Magnitude rule, for both responses, but at the .05 significance level. The Magnitude rule also gave faster RTs than did Curvilinearity considered alone at the .05 level. In addition, there was a significant interaction between type of response and sameness rule $(p<.05)$ due to the greater difference between RTs for the two responses with increasing overall difficulty. Once again, mean errors in general reflected these differences in RT.

The result of importance is that the cognitive dimension of Magnitude was processed faster than the other dimensions used as sameness rules, even though the Curvilinearity dimension is physical rather than cognitive. Thus, it is possible for the ordering of processing speeds not necessarily to reflect the ordering from physical to cognitive dimensions.

Physically Identical pairs. Table 6 shows mean RTs for each of the 10 stimulus pairs for each of the sameness rules, with data collapsed across order for the pairs that are different. For the Physically Identical pairs, there are no significant differences among the various stimuli, and there is little regularity in the speed of processing of particular stimuli from one sameness rule to another. 
Table 5

Simultaneous Presentation, Stimuli 3, 4, 6, 7: Mean RT (in Milliseconds) and Mean Errors per Trial for "Same" and "Different" Responses and Four Sameness Rules

\begin{tabular}{|c|c|c|c|c|c|c|c|c|c|c|}
\hline \multirow[b]{3}{*}{ Response } & \multicolumn{10}{|c|}{ Sameness Rule } \\
\hline & \multicolumn{2}{|c|}{ Physical } & \multicolumn{2}{|c|}{ Magnitude } & \multicolumn{2}{|c|}{ Oddness } & \multicolumn{2}{|c|}{ Curvilinearity } & \multicolumn{2}{|c|}{ Mean } \\
\hline & RT & Errors & RT & Errors & $\mathrm{RT}$ & Errors & RT & Errors & $\mathrm{RT}$ & Errors \\
\hline $\begin{array}{l}\text { "Same" } \\
\text { "Different" }\end{array}$ & $\begin{array}{l}385 \\
405\end{array}$ & $\begin{array}{l}.016 \\
.007\end{array}$ & $\begin{array}{l}448 \\
485\end{array}$ & $\begin{array}{l}.029 \\
.016\end{array}$ & $\begin{array}{l}474 \\
515\end{array}$ & $\begin{array}{l}.048 \\
.026\end{array}$ & $\begin{array}{l}484 \\
542\end{array}$ & $\begin{array}{l}.048 \\
.040\end{array}$ & $\begin{array}{l}448 \\
487\end{array}$ & $\begin{array}{l}.035 \\
.022\end{array}$ \\
\hline Mean & 395 & .012 & 467 & .022 & 494 & .037 & 513 & .044 & 467 & .029 \\
\hline
\end{tabular}

Table 6

Simultaneous Presentation, Stimuli 3, 4, 6, 7: Mean RT (in Milliseconds) for Each Stimulus Pair and Each Sameness Rule

\begin{tabular}{|c|c|c|c|c|}
\hline \multirow{2}{*}{$\begin{array}{l}\text { Stimulus } \\
\text { Pair }\end{array}$} & \multicolumn{4}{|c|}{ Sameness Rule } \\
\hline & Physical & Magnitude & Oddness & Curvilinearity \\
\hline & \multicolumn{4}{|c|}{ Physical Identity } \\
\hline $\begin{array}{ll}3 & 3 \\
4 & 4 \\
6 & 6 \\
7 & 7\end{array}$ & $\begin{array}{l}390^{*} \\
382^{*} \\
378^{*} \\
391^{*}\end{array}$ & $\begin{array}{l}425^{*} \\
406^{*} \\
416^{*} \\
416^{*}\end{array}$ & $\begin{array}{l}420^{*} \\
414^{*} \\
413^{*} \\
426^{*}\end{array}$ & $\begin{array}{l}417^{*} \\
423^{*} \\
417^{*} \\
412^{*}\end{array}$ \\
\hline & \multicolumn{4}{|c|}{ Same Magnitude } \\
\hline \multirow[t]{2}{*}{$\begin{array}{l}34 \\
67\end{array}$} & $\begin{array}{l}404 \\
393\end{array}$ & $\begin{array}{l}482^{*} \\
479^{*}\end{array}$ & $\begin{array}{l}489 \\
498\end{array}$ & $\begin{array}{l}505 \\
512\end{array}$ \\
\hline & \multicolumn{4}{|c|}{ Same Oddness } \\
\hline $\begin{array}{l}37 \\
46\end{array}$ & $\begin{array}{l}380 \\
414\end{array}$ & $\begin{array}{l}469 \\
499\end{array}$ & $\begin{array}{l}565^{*} \\
496^{*}\end{array}$ & $\begin{array}{l}572 \\
579\end{array}$ \\
\hline & \multicolumn{4}{|c|}{ Same Curvilinearity } \\
\hline $\begin{array}{l}36 \\
47\end{array}$ & $\begin{array}{l}428 \\
411\end{array}$ & $\begin{array}{l}487 \\
486\end{array}$ & $\begin{array}{l}553 \\
518\end{array}$ & $\begin{array}{l}543^{*} \\
557^{*}\end{array}$ \\
\hline
\end{tabular}

*"Same" responses.

For the different sameness rules, there is an overall statistically significant effect, but it is due entirely to the fact that the Physical Identity rule provides faster processing than the other rules, none of which is significantly different from the others. The relatively low RTs and the lack of effect of sameness rule for Physically Identical pairs again means that combining them with "same" responses for nonidentical pairs gives a somewhat unclear picture of the effect of response type and the interaction of that effect with the sameness rule. In an analysis that uses only nonidentical stimulus pairs, the mean RT for "same" is $520 \mathrm{msec}$, while that for "different" is $514 \mathrm{msec}$, an actual but statistically nonsignificant reversal of the usually found advantage for "same" responses. Furthermore, there was no significant interaction between type of response and sameness rule. Thus, in all three sets of data, the advantage for "same" responses is missing or minimal when Physically Identical stimuli are not included in the analysis.

Numerical distance. The most effective dimension in processing these numerals was Magnitude, which was used as a sameness rule by requiring "same" responses for the two low numbers or the two high numbers. There is, of course, an actual continuum of numerical distance across the entire set of four numerals. If the Magnitude dimension is so effective with these stimuli, it seems reasonable that the entire continuum should be reflected in performance, and certainly work on the symbolic distance effect for number (e.g., Moyer \& Landauer, 1967; also, more recently, Hinrichs, Yurko, \& Hu, 1981) would suggest such an effect.

The data show that, for the "same" response, an effect is operating over the entire range of numbers, regardless of the sameness rule. Figure 4 shows mean $\mathrm{RT}$ as a function of the numerical distance between pairs of letters that were called "same," plotted for each pair of stimuli that were actually different. Also plotted are mean RTs for the identical pairs for the Physical Identity and the other sameness rules, but averaged for all identical numeral pairs. The overall correlation for these data is .96 , a correlation that makes the importance of the effect quite clear. Of additional interest is an examination of the two sets of pairs for each sameness rule. For both Same Mag-

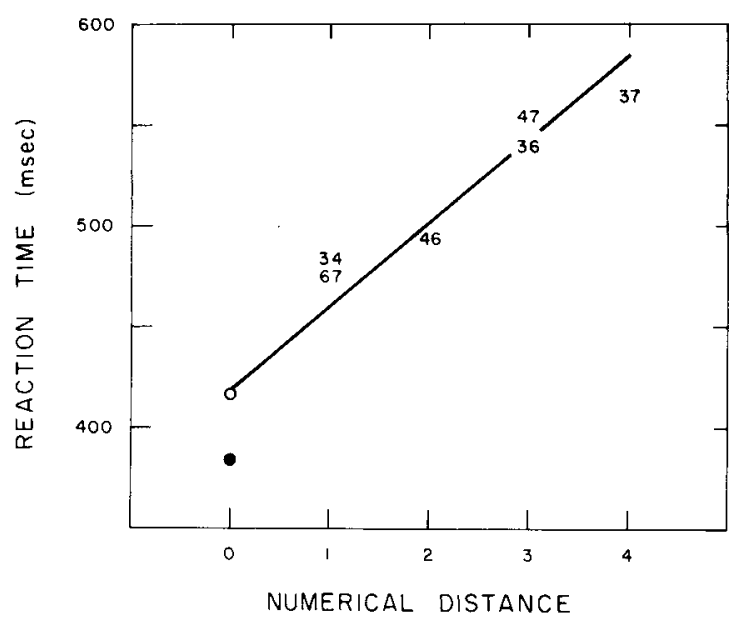

Figure 4. Stimuli 3, 4, 6, 7: Reaction time for "same" responses as a function of numerical distance. The specific stimulus pairs are plotted for numerical distances greater than 0 . At 0 distance, the closed circle is for the Physical Identity rule and the open circle is for all other rules. 
itude and Same Curvilinearity stimuli, the numerical difference between the two numerals of the two pairs is equal, and the differences in RT between the two pairs in each case is small. For the Oddness stimuli, the pair 37 has a difference of 4 , and the pair 46 has a difference of 2 , and RTs for these two pairs differ by $69 \mathrm{msec}$, placing them very properly on the regression line shown in Figure 4. The difference between these two pairs is very consistent across subjects, being shown by all eight.

Despite the importance of numerical distance on "same" responses, it has no consistent effect on "different" responses, for any of the sameness rules, given the expectation that a greater numerical distance should lead to faster "different" responses. In fact, the data for the six pairs of different stimuli for the Physical Identity rule for sameness lead to no usable solution for a discriminability scale, which the analogous data for Experiment 1 did. (There is actually a slight suggestion that the physical dimension of Curvilinearity is influencing "different" responses with the Physical Identity rule, since the two types of stimulus pairs that differ on the Curvilinearity dimension give slightly faster RTs, but data from other sameness rules do not confirm its role.)

Ease of processing. This failure to obtain a reasonable scale of discriminability is probably due to the fact that particular numeral pairs lead to greater ease of processing and that such ease of processing is interacting with or overriding any effects of either physical discriminability or discriminability based on numerical distance. The strongest evidence for the role of ease of processing comes from the fact that the Same Magnitude pairs, 34 and 67, are responded to as "different" with both the Oddness and the Curvilinearity rules much faster than are the other nonidentical stimulus pairs that require the "different" response. This difference is $42 \mathrm{msec}$ for the Oddness rule and $67 \mathrm{msec}$ for the Curvilinearity rule, and in each case the difference is statistically significant, with all eight subjects showing the effect.

This greater ease of processing for adjacent numerals was found earlier by Fitts and Switzer (1962) in an experiment requiring identification rather than comparison responses. As one illustration, RT to identify the numeral 2 was faster when it was paired with the numeral 1 as the alternative rather than with the numeral 7. As these authors demonstrated, and as the present experiments confirm, discriminability alone does not account for speed of a discrimination response: cognitive factors concerning properties of the particular stimuli to be discriminated play an important role as well, often sufficiently great to obscure any simple effects of discriminability.

Stimulus order effects show no consistent pattern, averaging $9 \mathrm{msec}$ for the 24 different stimulus pairs, with a standard deviation of $9 \mathrm{msec}$ and a maximum difference of $35 \mathrm{msec}$. These data are much like those obtained with simultaneous presentation in Experiment 1. One point of special interest is that there is no processing advantage for a pair of stimuli that occurred in their natural order.

\section{Summary and Conclusions}

(1) A cognitive dimension of numbers (Magnitude) can be processed faster than a clearly physical dimension (Curvilinearity), even though Experiment 1 had shown the curved-straight difference (translated from System as a dimension) to be more effective than the Name dimension. There is therefore no immutable processing order from most physical to most cognitive.

(2) While numerical distance accounted for essentially all of the differences in speed of "same" responses, familiar pairs, such as adjacent numbers, facilitated processing of both "different" and "same" responses. Thus, in confirmation of the results of Experiment 1, some stimulus pairs are simply processed more easily than others, leading to fast RTs to both "same" and "different" responses, and therefore not reflecting discriminability differences.

\section{GENERAL DISCUSSION}

Discussion of most of the findings of these experiments was included within the sections for each experiment. There are, however, three topics that these experiments bear on and that need new or further comment.

\section{Selective Attention}

Most of the research that has been done with sets of stimuli dimensionally defined has been concerned with dimensional interactions, especially in the work of Garner (1974) and his colleagues. A major distinction has been made between integral and separable dimensions, and one of the experimental outcomes used to differentiate these dimensional interactions has been whether stimuli can be classified with no interference from irrelevant variations in a second dimension. If subjects can selectively attend to one dimension while another varies irrelevantly, then the dimensions are considered separable; if such selective attention is not possible, then the dimensions are integral. Sometimes experimental results have shown that the ability to attend to dimensions selectively is asymmetrical (see Garner, 1974, and in press, for a review), such that one dimension's irrelevant variation interferes with classification by the other, but not the reverse. Such results have frequently been interpreted as indicating a difference in levels of processing. Wood (1975), for example, suggested such an interpretation for the physical dimension of pitch combined with the phonetic dimension of consonant. This interpretation fits naturally the distinction between physical and cognitive dimensions, with the 
reasonable expectation that a physical dimension (e.g., pitch) should interfere with classification of a cognitive dimension (e.g., consonant), but not the converse.

Perfect selective attention to a dimension in the same-different paradigm would lead to no greater processing time for nonidentical stimuli differing in a dimension than for identical stimuli, under a given rule for sameness. Thus, it might have been expected that when the physical dimension of Length was the rule for sameness in Experiment 1, responses to those stimulus pairs having the Same Length would be as fast as to Physically Identical stimuli. No such result was obtained for any dimension in either experiment, so there was no clear evidence that selective attention was possible for any of these dimensions and thus that any pair of dimensions was either symmetrically or asymmetrically separable. Before interpreting this result in a strong form, however, it should be pointed out that recent research by Santee and Egeth (1980) failed to show separability of dimensions with the same-different procedure even for dimensions that had shown separability, as evidenced by selective attention in a classification task. It is thus not clear how classification and comparison methods provide evidence concerning dimensional interaction.

\section{Discriminability and Ease of Processing}

In order to interpret an asymmetric interference effect as a difference in processing levels (and the present experiments did show asymmetry, since not all dimensions were processed equally fast), it is logically necessary, as Garner (in press) has emphasized, that there be control conditions that demonstrate equal discriminability of the dimensions, because a simple difference in discriminability of dimensions operating at the same processing level would show such an asymmetric result. Such controls for discriminability in the present experiments come from the RTs for "different" responses with Physical Identity as the sameness rule, since all nonidentical pairs require a "different" response with that sameness rule. In Experiment 1 especially, these measures did not show equal discriminability of the dimensions, and in fact showed discriminability differences for the three dimensions that were ordered exactly as the overall difficulty of processing each dimension as the rule for sameness.

However, even though these results suggest that no easy interpretation relying on levels of processing is possible, they also show that the concept that particular stimuli or stimulus pairs are more or less easy to process than others are is valid and important in understanding the differences between physical and cognitive dimensions. In both experiments, some stimulus pairs were consistently easy to process regardless of whether a "same" or a "different" response was required, a result that would not be expected if discriminability were the only factor deter- mining speed of processing. In this respect, the results are like those of Sebrechts and Garner (1981), who showed that configurally good patterns were processed faster than poor patterns with both "same" and "different" responses.

On the other hand, insofar as discriminability does seem to be a determining factor in speed of processing, Experiment 1 indicated that it was more so for the Physical Identity rule for sameness or for the physical dimension of Length. Even in the latter case, however, some reversals of RT that should have occurred when the response was changed from "different" to "same" did not do so. Podgorny and Garner (1979) did find that, with a comparison task for uppercase letters, the relation between RTs for different letters did change when the response was changed from "different" to "same." The best conclusion seems to be that discriminability is an important factor in processing speed if processing of relatively straightforward physical properties is required, although even in such cases strong cognitive factors can influence processing and alter the simple relation between discriminability and $\mathrm{RT}$.

\section{Stage Conceptions of Processing}

The present data do not lend themselves to an interpretation in terms of simple discrete processing stages. The fact that a cognitive dimension can be processed faster than a fairly adequate physical dimension, as in Experiment 2, is excellent evidence against such simple stage conceptions. The present results do fit rather comfortably into Posner's (1978) idea of isolable systems, in which parallel processing of different "levels" goes on after initial processing of physical properties. There are also other conceptions of stage processing, such as those of McClelland (1979) and Taylor (1976), that might be adapted to the present data.

However, Garner (1980) has argued that it is probably more effective to seek clarification of functions rather than location of stages, and the present data do indicate that fairly detailed data analyses can clarify how stimuli are processed. The fact that a name can be used as a processing mechanism but length apparently cannot is not a result that differentiates stages, but it most definitely differentiates function. Similarly, the fact that numerical order and distance dominate processing of numerals in at least the Arabic system is not a result indicating stage differences, but one indicating functional differences. The search for function rather than stages will be effective, at least with some types of stimulus dimensions.

\section{REFERENCES}

Broadbent, D. E. Stimulus set and response set: Two kinds of selective attention. In D. I. Mostofsky (Ed.), Attention: Contemporary theory and analysis. New York: Appleton-CenturyCrofts, 1970. 
Fitts, P. M., \& Switzer, G. Cognitive aspects of information processing: I. The familiarity of S-R sets and subsets. Journal of Experimental Psychology, 1962, 63, 321-329.

GARNER, W. R. The processing of information and structure. Potomac, Md: Erlbaum, 1974.

GaRNER, W. R. Adequate and inadequate stimulus information: Comments on "Some considerations of two alleged kinds of selective attention" by Keren. Journal of Experimental Psychology: General, 1976, 4, 375-377.

Garner, W. R. Association lecture: Functional aspects of information processing. In R. S. Nickerson (Ed.), Attention and performance VIII. Hillsdale, N.J: Erlbaum, 1980.

Garner, W. R. Asymmetric interactions of stimulus dimensions in perceptual information processing. In T. J. Tighe \& B. E. Shepp (Eds.), Interactions: Perception, cognition, \& development: A second Dartmouth multi-perspective conference. Hillsdale, N.J: Erlbaum, in press.

Garner, W. R., \& Felfoldy, G. L. Integrality of stimulus dimensions in various types of information processing. Cognitive Psychology, 1970, 1, 225-241.

GotTWALD, R. L., \& GARNER, W. R. Effects of focusing strategy on speeded classification with grouping, filtering, and condensation tasks. Perception \& Psychophysics, 1972, 11, 179-182.

Hinrichs, J. V., Yurko, D. S., \& Hu, J.-M. Two-digit number comparison: Use of place information. Journal of Experimental Psychology: Human Perception and Performance, 1981, 7, 890-901.

KEREN, G. Some considerations of two alleged kinds of selective attention. Journal of Experimental Psychology: General, 1976, 105, 349-374.

McClelland, J. L. On the time relations of mental processes: An examination of systems of processes in cascade. Psychological Review, 1979, 86, 287-330.

Moyer, R. S., \& Landauer, T. K. Determinants of reaction time for digit inequality judgments. Nature, 1967, 215, 1519-1520.
Nickerson, R. S. Binary-classification reaction time: A review of some studies of human information-processing capabilities. Psychonomic Monograph Supplements, 1972, 4(17, Whole No. 65).

Nickerson, R. S. On the time it takes to tell things apart. In J. Requin (Ed.), Attention and performance VII. Hillsdale, N.J: Erlbaum, 1978

Podgonny, P., \& Garner, W. R. Reaction time as a measure of inter- and intraobject visual similarity: Letters of the alphabet. Perception \& Psychophysics, 1979, 26, 37-52.

Posner, M. I. Chronometric exploration of mind. Hillsdale, N.J: Erlbaum, 1978.

Posner, M. I., \& Mitchell, R. F. Chronometric analysis of classification. Psychological Review, 1967, 74, 392-409.

Proctor, R. W. A unified theory for matching-task phenomena. Psychological Review, 1981, 88, 291-326.

Santee, J. L., \& Egeth, H. E. Selective attention in the speeded classification and comparison of multidimensional stimuli. Perception \& Psychophysics, 1980, 28, 191-204.

Sebrechts, M. M., \& Garner, W. R. Stimulus-specific processing consequences of pattern goodness. Memory \& Cognition, $1981,9,41-49$.

TAYlor, D. A. Stage analysis of reaction time. Psychological Bulletin, 1976, 83, 161-191.

TVERsky, B. Pictorial and verbal encoding in a short-term memory task. Perception \& Psychophysics, 1969, 6, 225-233.

Wood, C. C. Auditory and phonetic levels of processing in speech perception: Neurophysiological and information-processing analyses. Journal of Experimental Psychology: Human Perception and Performance, 1975, 1, 3-20.

(Manuscript received January 12, 1982; accepted for publication February 19, 1982.) 